\title{
Understanding the Relationship Between Air Exposure, Electron Dose and Beam Damage in Solid Electrolyte Materails
}

Eric Stach ${ }^{1}$, James Horwath ${ }^{1}$, Nikhilendra Singh $^{2}$, Timothy Arthur ${ }^{2}$, Daan Hein Alsem ${ }^{3}$ and Norman Salmon $^{3}$

${ }^{1}$ University of Pennsylvania, Philadelphia, Pennsylvania, United States, ${ }^{2}$ Toyota Research Institute of North America, Ann Arbor, Michigan, United States, ${ }^{3}$ Hummingbird Scientific, Lacey, Washington, United States

Solid state batteries offer significant potential advantages over existing technologies in electrical vehicle applications. Because they are not generally flammable, they offer potential safety benefits and their competitive energy density provides the ability to create more compact battery packs. Of particular importance in understanding how to optimize these systems is quantitively determining how lithium transports through the solid electrolyte and correlating quantitative electrochemical data to any structural transformations that occur in the system.

However, solid electrolytes are known to be highly sensitive to moisture. This significantly complicates our ability to utilize transmission electron microscopy to make these correlations, as common sample preparation and transfer approaches usually lead to unwanted air exposure. In this presentation, we will discuss our development of methods for characterizing these samples following careful control over exposure to moisture and at appropriate levels of both dose and dose rate.

In the first portion of the presentation, we will describe the development of a dedicated vacuum transfer holder that allows us to prepare samples in a dry environment (i.e. a glove box) and transfer the samples without air exposure into the TEM. Through a combination of controlled dose imaging and spectroscopic mapping using energy dispersive $\mathrm{x}$-ray spectroscopy we will show that this leads to a dramatically improved ability to obtain spectroscopic data without damaging the sample. These studies provide a baseline, ex-situ method to characterize the effects of lithium transport in these systems.

Thereafter, we will describe how we can use operando TEM methods to correlate electrochemical data and structural transformations, again following careful control over air exposure and dose and dose rate. In particular, we take advantage of a commercially available 'nanomanipulation' holder that allows us to create highly local electrical contacts to the sample and perform cyclic voltammograms concomitant with imaging and spectroscopic characterization(Figure 1). Throughout the presentation we will focus on the necessary experimental protocols to produce repeatable, artifact free results between experiments [1]. 

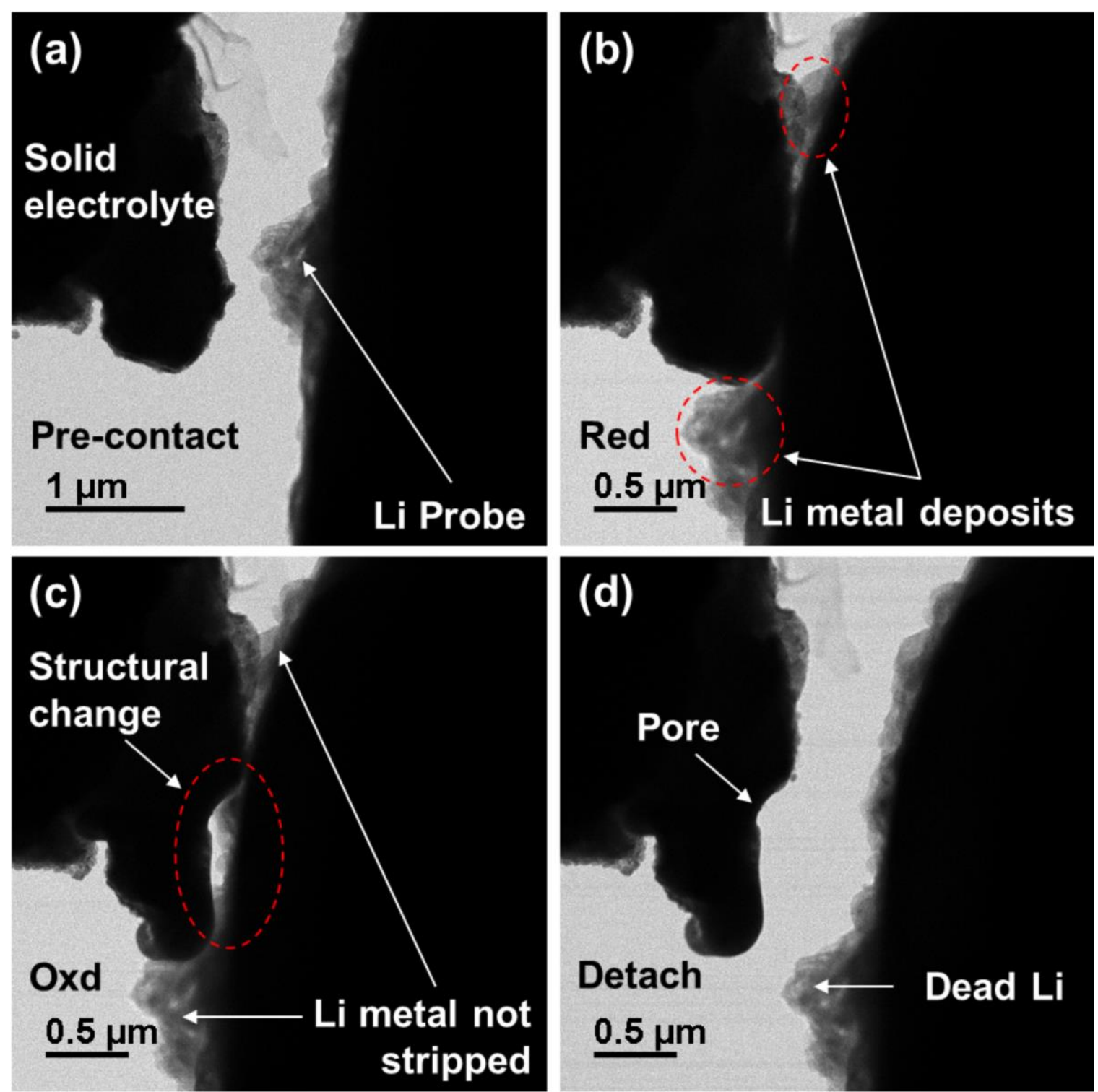

Figure 1. Changes in the structure of a solid electrolyte observed during operando biasing nanomanipulation analysis. (a) the pristine solid electrolyte and lithium metal materials, (b) the deposition of lithium metal upon contact and the application of a reductive bias, (c) structural changes in the solid electrolyte observed during the stripping phase of lithium metal during an applied oxidative bias, and (d) retention of the structural change upon detachment of the lithium and solid electrolyte.

References

1. This research used resources of the Center for Functional Nanomaterials, which is a U.S. DOE Office of Science Facility, at Brookhaven National Laboratory under Contract No. DE- SC0012704. 